\title{
Pathophysiology of Nonalcoholic Steatohepatitis
}

\author{
SUMIO WATANABE* \\ * Department of Gastroenterology, Juntendo University Faculty of Medicine, Tokyo, Japan
}

\begin{abstract}
Nonalcoholic steatohepatitis (NASH), occurs frequently with diabetes mellitus and obesity, contributes to insulin resistance, can develop cirrhosis or even hepatocellular carcinoma (HCC). Although NASH has become more common, its underlying mechanism is still not clear and effective therapy has not been established. Currently, it has been recognized that the multiple parallel factors, including genetic differences, insulin resistance, lipotoxicity and dysbiosis act synergistically in genetically in pathogenesis of NASH. Impaired autophagy parallel to excess lipid supply might explain the progression of NASH to HCC. Liver-specific knockout mice of phosphatase and tensin homolog deleted on chromosome ten (PTEN) develops steatohepatitis followed by HCC, suggesting a potential role for this molecule in development and progression of NASH.
\end{abstract}

Key words: nonalcoholic fatty liver disease (NAFLD), autophagy, lipotoxicity, phosphatase and tensin homolog deleted on chromosome ten (PTEN), innate immunity

\section{Introduction}

In 1980, Ludwig and colleagues named nonalcoholic steatohepatitis (NASH) in individuals whose liver biopsies showed findings includes steatosis, inflammation, Mallory-Denk hyaline bodies and perisinusoidal/pericellular fibrosis, which are similar to alcoholic liver injury in the absence of significant alcohol intake ${ }^{1)}$ (Figure-1). Subsequently, nonalcoholic fatty liver disease (NAFLD) has been used as a general name for conditions ranging from non-alcoholic fatty liver (NAFL), which is the non-progressive form, to NASH, the progressive form (Figure-2). Approximately 10\% of patients with NAFLD have NASH, and $32-53 \%$ of patients with NASH will progress fibrosis, which leads to cirrhosis. Although research for NAFLD/ NASH is the constantly advancing field ${ }^{2)}$, the pathogenesis of NASH is still not fully elucidated and there is no established therapy for NASH. Here, I review about outlines of some topics about pathophysiology of NASH.

\section{Practice guidelines for NAFLD/NASH}

Over the past 2 decades, prevalent rate of NASH has been rapidly increased in development countries and has become a common disease ${ }^{3)}$. The prevalence of NASH-related cirrhosis has been reported as high as 2.5-fold increases in 2009-2012 compared to 1999-2002 in the United States ${ }^{4)}$. Number of studies of NAFLD/NASH increases continuously with its high prevalence worldwide (Figure-3). The Japanese practice guideline for NAFLD/NASH, established by the Japanese Society of Gastroenterology in cooperation with

\section{Sumio Watanabe}

Department of Gastroenterology, Juntendo University Faculty of Medicine

2-1-1 Hongo, Bunkyo-ku, Tokyo 113-8421, Japan

TEL: +81-3-3813-3111 FAX: +81-3-3813-8862 E-mail: sumio@juntendo.ac.jp

341st Triannual Meeting of the Juntendo Medical Society: Farewell Lectures of Retiring Professors [Held on Mar. 29, 2017]

〔Received June 7, 2017] [Accepted June 26, 2017〕

Copyright (C) 2017 The Juntendo Medical Society. This is an open access article distributed under the terms of Creative Commons Attribution License (CC BY), which permits unrestricted use, distribution, and reproduction in any medium, provided the original source is properly credited. doi: $10.14789 / j m j .63 .230$ 


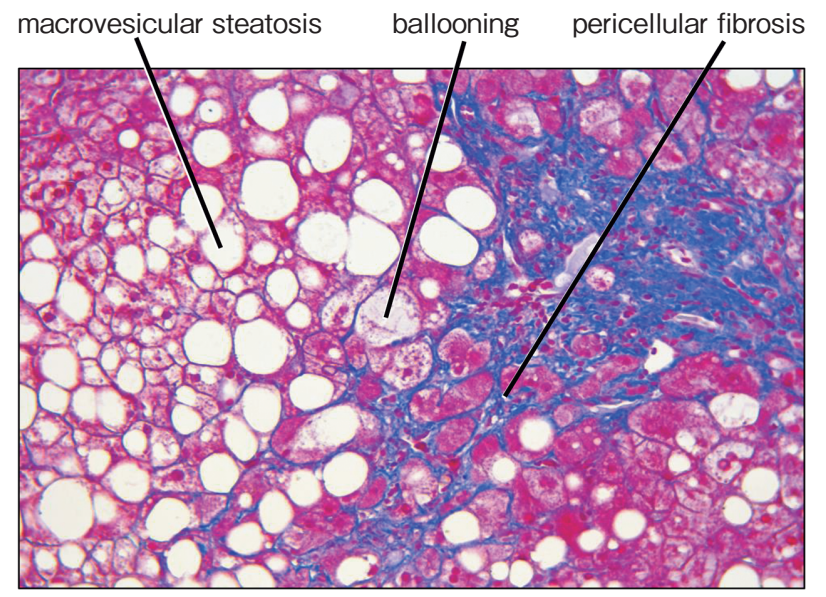

67y.o Male NASH grade 2, stage 3

Figure-1 Pathological findings of NASH

This image shows typical pathological findings of NASH, which are macrovesicular steatosis, hepatocyte ballooning, and perivenular/ pericellular fibrosis.

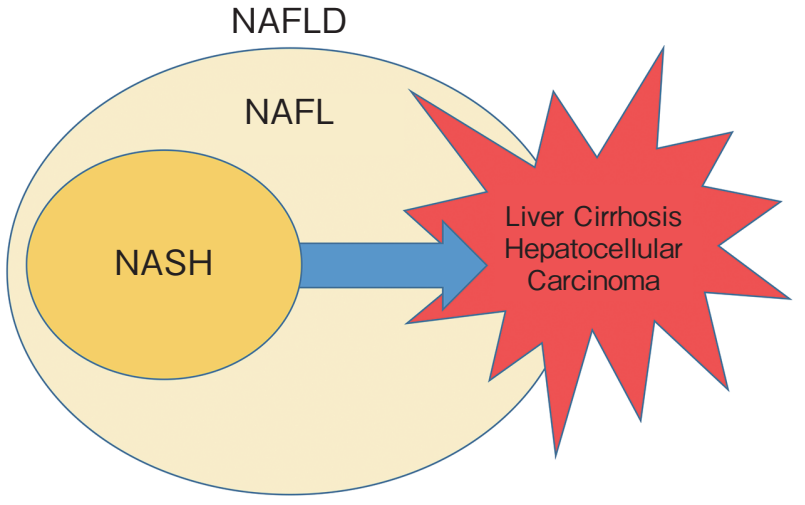

Figure-2 The definition of NAFLD/NASH

NAFLD is categorized histologically into nonalcoholic fatty liver (NAFL) and nonalcoholic steatohepatitis (NASH). NAFL is defined as hepatic steatosis with no evidence of hepatocellular injury in the form of hepatocyte ballooning. NASH is defined as the presence of hepatic steatosis and inflammation with hepatocyte injury (ballooning) with or without fibrosis. NASH may progress to cirrhosis and/or hepatocellular carcinoma.

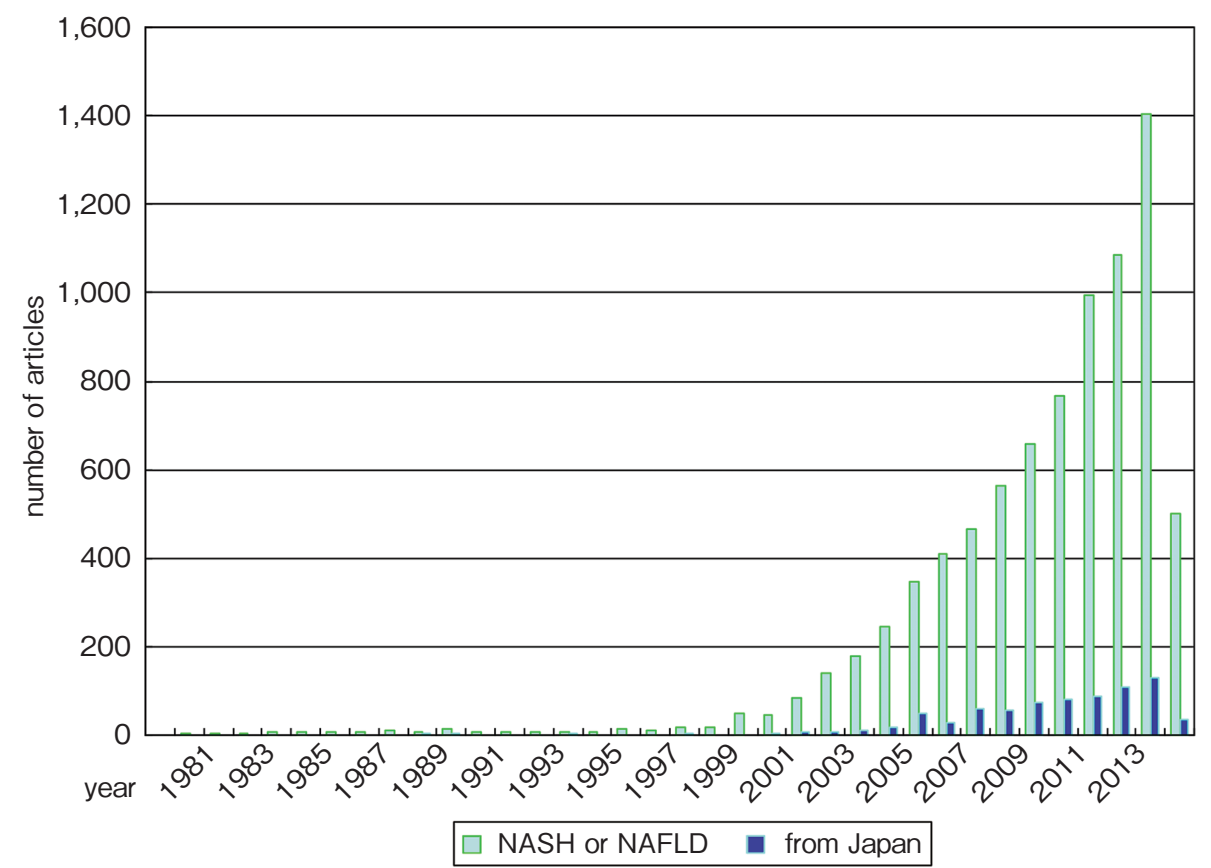

Figure-3 The number of articles related to NAFLD/NASH in PubMed (Medline) published every year Green bar: Articles including the word "NAFLD" or "NASH" published from 1980 to 2014 was searched in PubMed and the number of them each year was plotted. Blue bar: The number of articles submitted from Japan for same period was also plotted.

The Japan Society of Hepatology, was published in 2014, and English version was also published in $2015^{5)}$ (6) , in which I took on the position of chairman of the production committee.

\section{The impact of liver-specific PTEN knock-out mouse model}

Although there are several animal models of
NAFLD/NASH, there is no existing model exhibits the entire NAFLD phenotype as encountered in clinical practice. Especially, the establishment of animal models for NASH with stably developing hepatocellular carcinoma (HCC) is tremendously valuable to prolong survival and improve the prognosis of patients with NAFLD/NASH. The phosphatase and tensin homolog deleted on chromosome ten (PTEN) negatively regulates the 
A

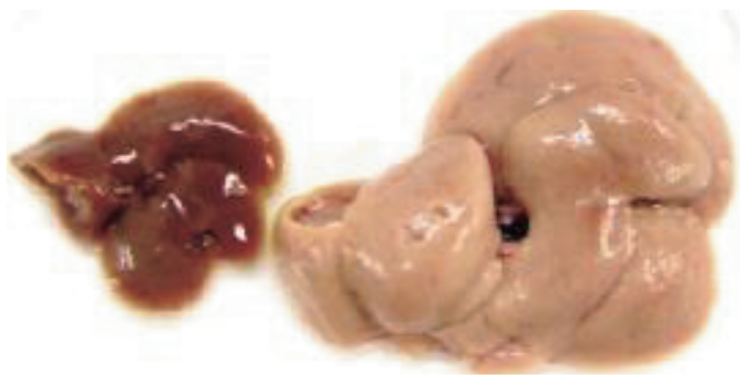

B
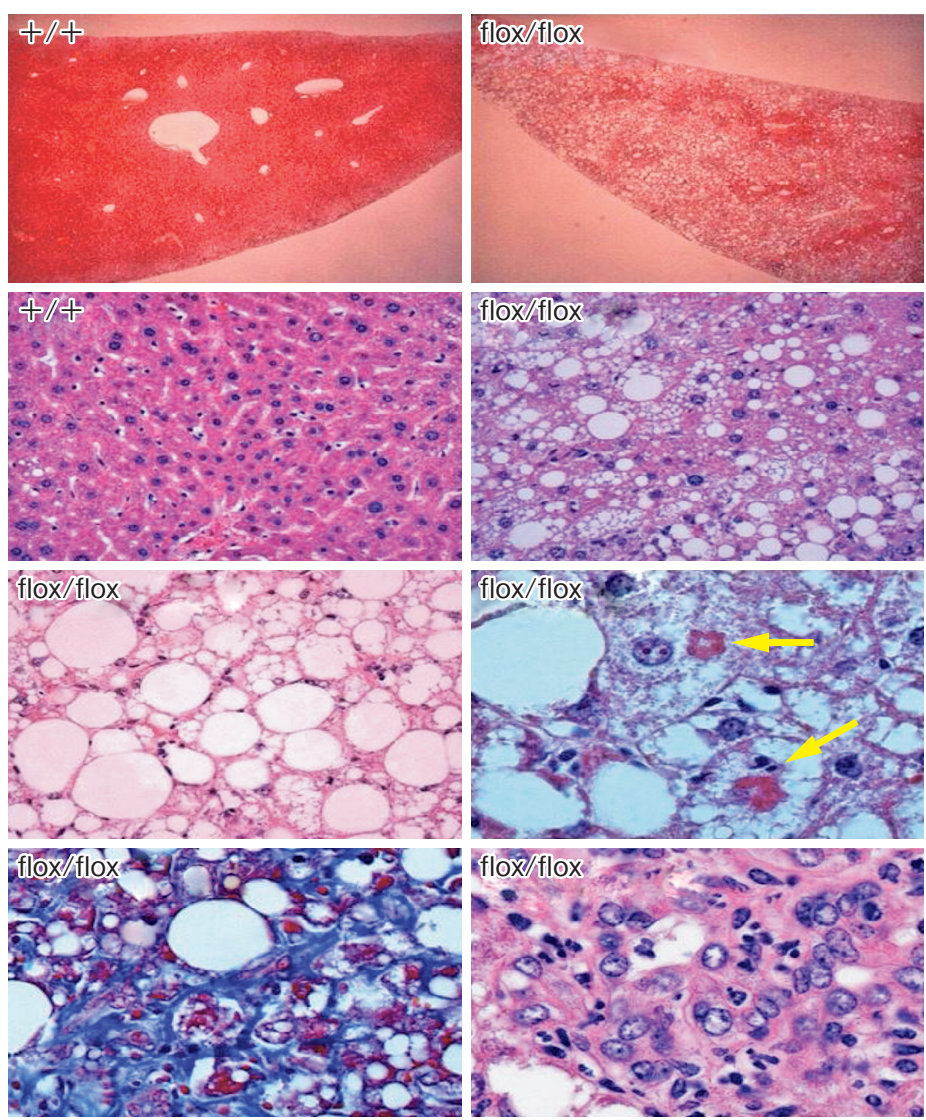

C
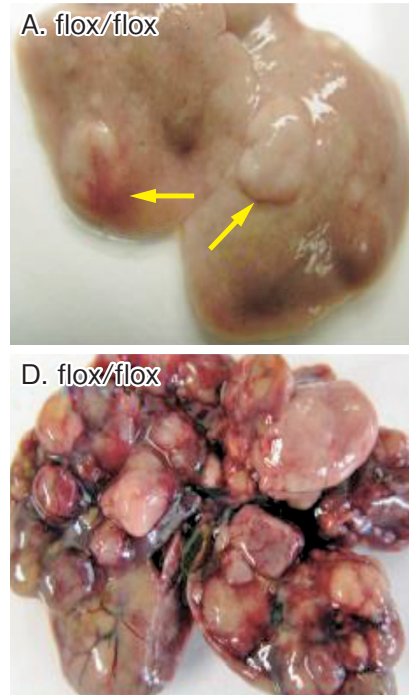
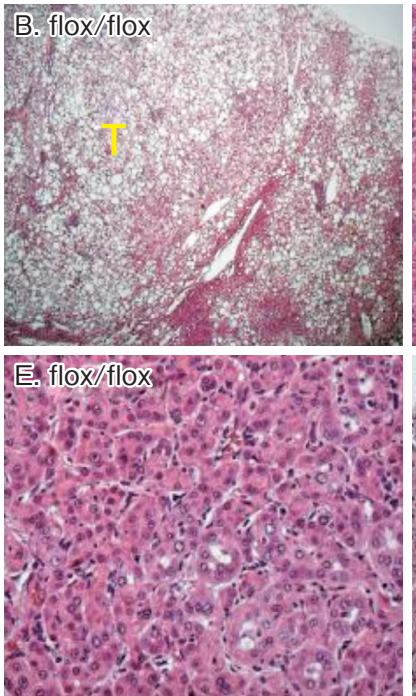

40-44 weeks
Figure-4 Pten-knock out mice developed steatohepatitis followed by hepatic fibrosis and HCC progression

A: Liver of control mice (left) and Pten-knock out mice (right).

B: Microscopic findings of liver of control mice (left) and Pten-knock out mice at 40-44 weeks old (right).

C: Liver of control mice (upper) and Pten-knock out mice (lower) at 74-78 weeks.

(Republished with permission of American Society for Clinical Investigation from Hepatocyte-specific Pten Deficiency results in steatohepatitis and hepatocellular carcinomas, Horie Y, et al., volume 113, 2004 ${ }^{7)}$; permission conveyed through Copyright Clearance Center. Inc.) 
phosphoinositol-3-kinase (PI3k)/AKT signaling pathway by dephosphorylating PIP3. We examined hepatocyte-specific Pten deficient (Pten knock out) mice, and demonstrated that Pten KO mice develops steatohepatitis strikingly similar to human NASH. Our data demonstrated that hepatic deficiency of Pten activates lipogenic signals such as the PI3K-PKB/Akt-Foxo1-PPAR $r$ pathway and PI3K-mediated SREBP1c induction. Moreover, 47\% of livers of Pten KO mice developed liver cell adenomas by 44 weeks of age and $66 \%$ of livers showed hepatocellular carcinoma by $74-78$ weeks of age. Therefore, Pten KO mice are a powerful animal model of NASH and NASH-related tumorigenesis $(\text { Figure }-4)^{7)}$, and it is expected that this model contribute to more development of research on NAFLD/NASH.

\section{Multiple parallel hits hypothesis and lipotoxicity}

A "two-hit hypothesis" has been proposed to explain the progression from NAFL, described the disease state at that time as "simple steatosis", to
NASH in $1998^{8)}$. The 'first hit', hepatic triglyceride accumulation, or steatosis, increases susceptibility of the liver to injury mediated by 'second hits', such as inflammatory cytokines/adipokines, mitochondrial dysfunction and oxidative stress, which in turn lead to steatohepatitis and/or fibrosis. This theory prevailed and become recognized by the world; however, the theory for explaining the development of NASH is now replaced by the 'multiple parallel hits' hypothesis, as knowledge of the interplay between insulin resistance, free fatty acids, adipokines, pathogenic factors from microbiota, and other less recognized factors have increased ${ }^{9)}$. In this hypothesis, a number of diverse parallel processes including adipose tissue-derived signals, gut barrier function, genetic factors, endoplasmic reticulum (ER) stress, and related signaling networks might contribute to the development of NASH. Especially, circulating lipid plays a major role in the development of NAFLD/NASH. Hepatic steatosis results from a disparity between lipid supply (from circulating lipid uptake and/or de novo lipogenesis) and lipid disposal (via fatty acid oxidation and/or

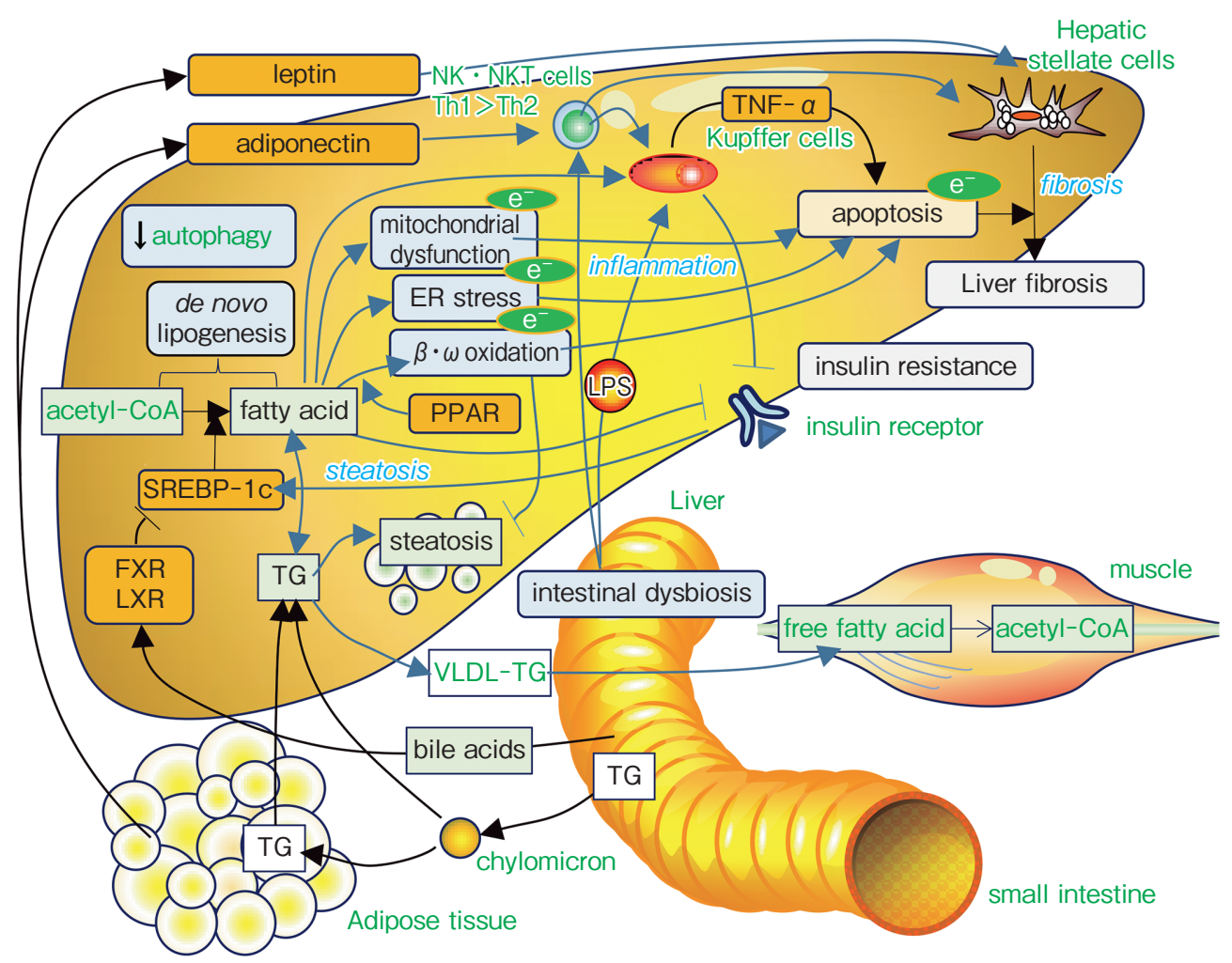

Figure-5 The relationship of fatty acid synthesis and lipid circulation with pathophysiology of NASH Increase of hepatic de novo lipogenesis and free fatty acids (FFAs) delivery from extrahepatic organs to the liver causes hepatic lipotoxicity. In parallel, hepatic oxidative stress/inflammation, and endoplasmic reticulum (ER) stress, changes in the profile of adipokines and cytokine, dysbiosis of gut microbiota promotes the development of NASH. 
production VLDL) in the liver. The overflow of free fatty acids (FFAs) coming from intestine and lipolysis of adipose tissue causes not only steatosis but also inflammation and fibrosis through enhancement of several physicochemical stress (Figure-5). We have reported that the intake of trans-fatty acid exacerbates hepatocyte of apoptosis through increase of ER stress and oxidative stress in mouse model ${ }^{10)}$. Regulation of lipid metabolism possibly a target for the therapy of NAFLD/NASH ${ }^{11}$.

\section{The role of innate immunity on NAFLD/NASH}

In recent years, the role of the innate immune response in NAFLD has been focus of intense research. Activation and recruitment of immune cells by local signals or signals derived from the adipose tissue or the gut, related to changes in bacterial translocation, may promote the inflammatory response leading to cell injury and death, thus promoting NAFLD disease progression. Originally, bacterial translocation and changes in the intestinal microbiome was considered to be associated with alcoholic liver disease. In alcoholic liver disease, passage of pathogen-associated molecular patterns (PAMPs) such as lipopolysaccharide trigger intracellular signaling cascades which activates downstream transcription and expression of a variety of genes involved in the immune and inflammatory host response. Innate immunity is contributed to both of hepatic inflammation and fibrosis ${ }^{12}$. Similar mechanism is involved in pathogenesis of NAFLD/NASH (Figure-6). We have reported the altered expression and function of hepatic natural killer T (NKT) cells in obese and diabetic KK- $\mathrm{A}^{\mathrm{y}}$ mice ${ }^{13)}$ and glycine prevents steatohepatitis in the mice through normalization of hepatic innate immune responses involving NKT cells and M2 transformation of Kupffer cells ${ }^{14)}$.

Adiponectin and leptin, which are secreted by adipose tissue, have been considered to play important roles in the progression of NAFLD/ NASH. Adiponectin has been shown to be protective against lipopolysaccharide-induced liver injury and hepatic fibrogenesis in $\mathrm{CCl}_{4}$-inudced liver fibrosis model. Diabetic KK- $\mathrm{A}^{\mathrm{y}}$ mice, which present phenotypes resembling metabolic syndrome in humans, lack physiological upregulation of adiponectin production caused by methionine-choline deficient (MCD) diet and develop severe steatohepatitis compared with wild mice ${ }^{15)}$. Additionally, several studies have shown that adiponectin exerts anti-inflammatory and antifibrogenic effects on the liver. In contrast, human study has shown that serum leptin levels are increased in patients with obese people. Leptin upregulates proinflammatory cytokines, which are associated with insulin resistance and type 2 diabetes mellitus. It has been reported that leptin/adiponectin imbalance was associated with increased waist circumference and

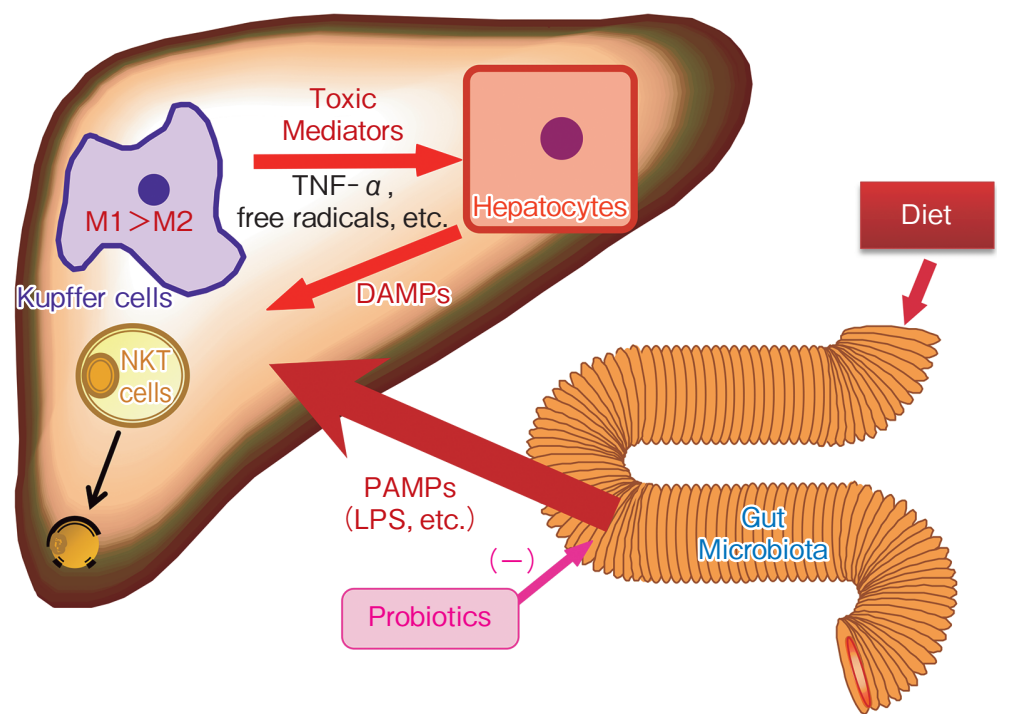

Figure-6 Activation of innate immunity in development of NASH Intestinal dysbiosis leads to increased translocation of pathogen associated molecular patterns (PAMPs) to liver. Not only PAMPs but also damage-associated molecular patterns (DAMPs) cause sterile inflammation in liver. 


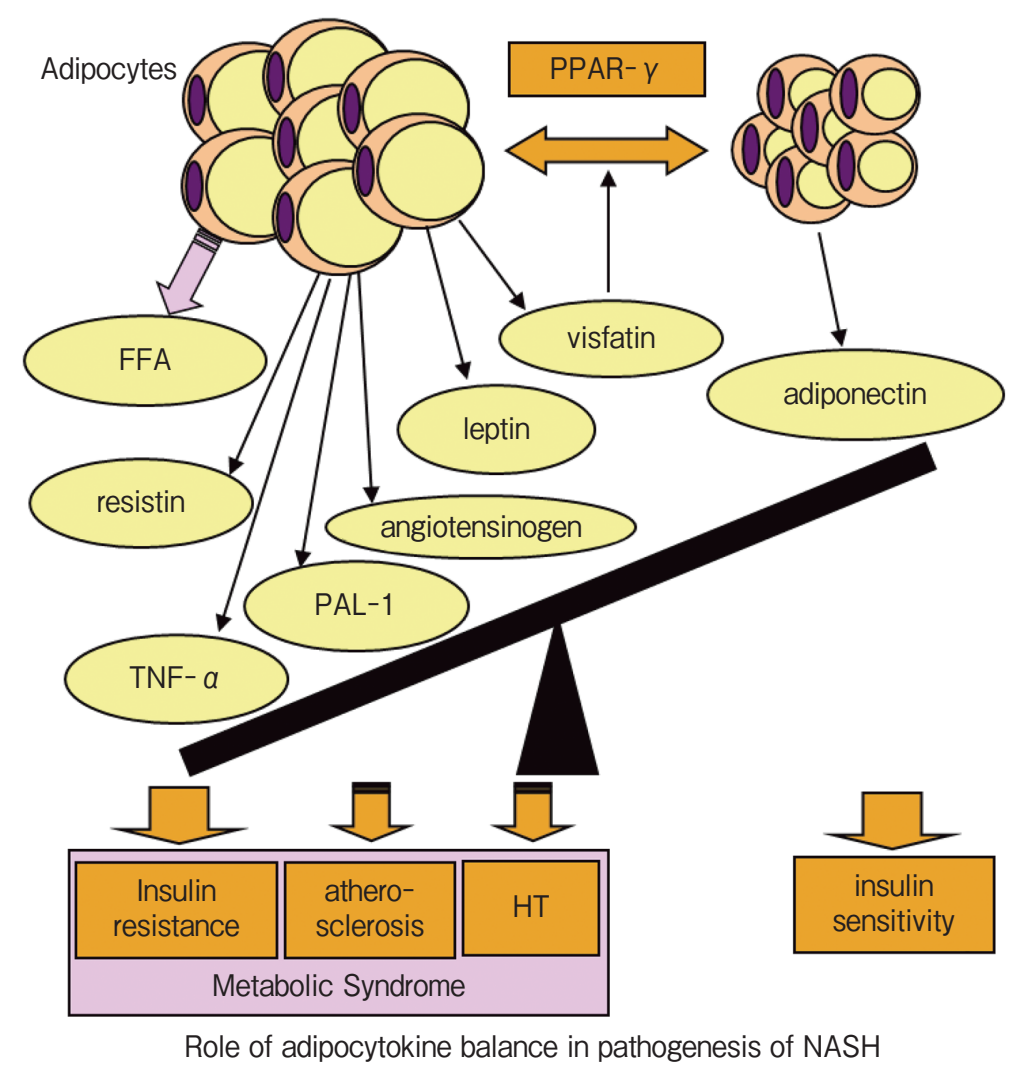

Figure-7 The role of adipocytokine balance on insulin resistance and metabolic syndrome Excessive enlargement of adipocytes due to lipid overloading alters adipokine balance and increases release of free fatty acids (FFAs), which contribute to the development of insulin resistance and metabolic syndrome-related diseases.

a decreased vascular response to acetylcholine and increased vasoconstriction due to angiotensin II in type 2 diabetes mellitus and cardiovascular diseases ${ }^{16)}$. Similarly, leptin/adiponectin imbalance may be contributed to the development of, and NAFLD through upregulation of inflammatory cytokines, impaired insulin sensitivity, and dysregulation of other metabolic factors (Figure-7). I and my collaborators have reported that obesityinduced leptin plays a crucial role in NASH progression via enhanced responsivity to endotoxin in mouse model ${ }^{17)}$.

\section{Autophagy dysregulation in pathogenesis of NASH}

Autophagy, or cellular self-digestion, is a catabolic process that targets cell constituents including damaged organelles, unfolded proteins, and intracellular pathogens to lysosomes for degradation. Autophagy is a mechanism involved in cellular homeostasis under basal and stressed conditions delivering cytoplasmic content to the lysosomes for degradation to macronutrients. We have reported that autophagy hepatic steatosis impairs autophagic proteolysis using obese and diabetic ob/ob mice. Whereas increased accumulation of autophagosome is observed, clearance of autolysosomes was blunted in hepatocytes from ob/ob mice, and proteinase activity of cathepsin $\mathrm{B}$ and $\mathrm{L}$ in autolysosomes and cathepsin $\mathrm{B}$ and $\mathrm{L}$ expression of liver were suppressed in ob/ob mice. These findings indicate that lipid accumulation blunts autophagic proteolysis via impairment of autophagosomal acidification and cathepsin expression ${ }^{18}$. Indeed, we have also reported that the number of autophagic vesicles in hepatocytes was increased in NAFLD patients ${ }^{19}$. Additionally, our collaborators have demonstrated that liver-specific autophagydeficient mice harbor adenomas linked to both the formation of $\mathrm{p} 62^{-}$and Keap1-positive cellular aggregates and induction of Nrf2 targets (Figure-8). These results suggest that dysregulation of autophagy is involved in the development and progression of NAFLD/NASH ${ }^{20}$. 

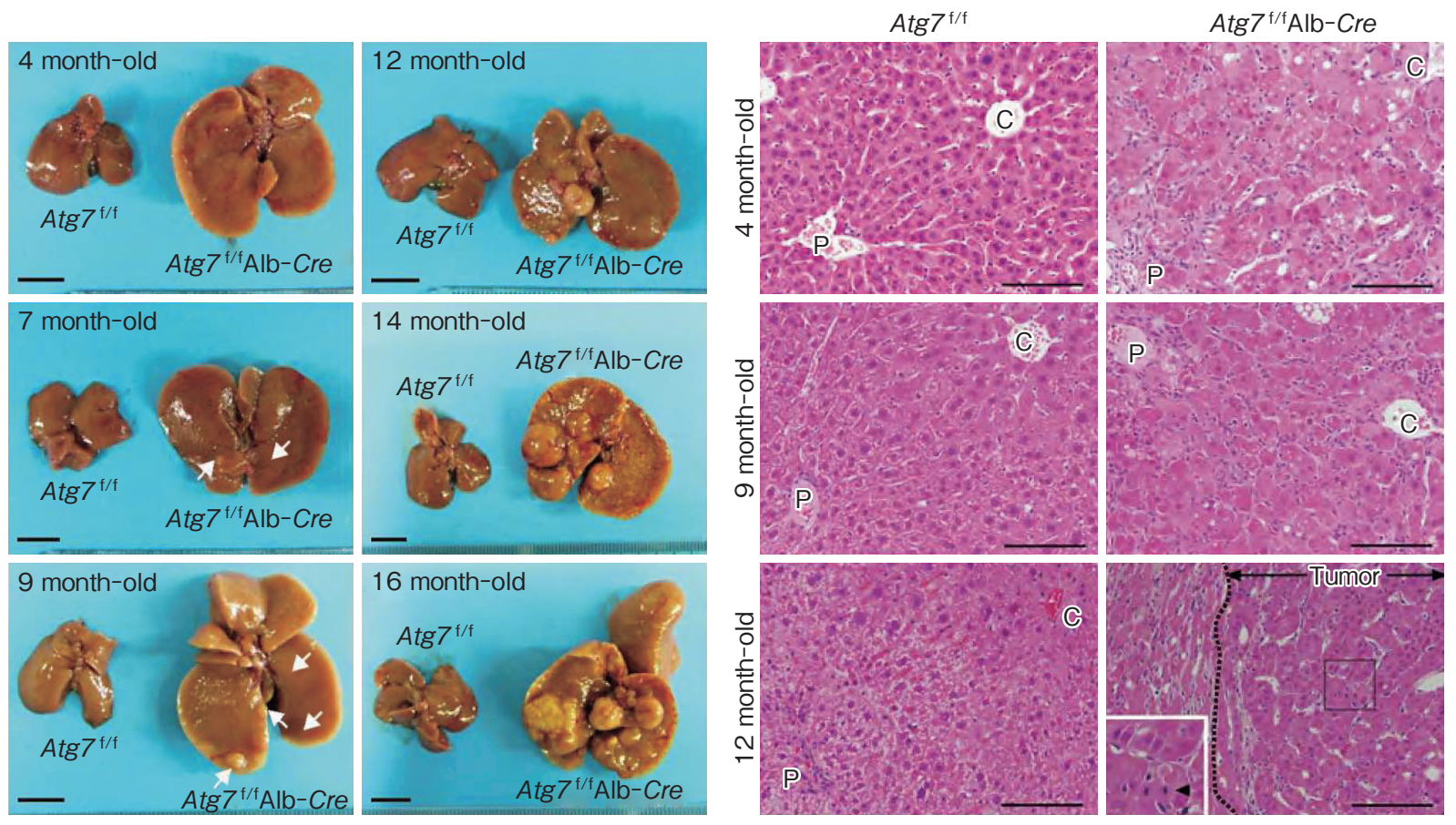

Figure-8 Inhibition of autophagy causes hepatocarcinogenesis

Liver-specific Atg7-deficient mice (Atg7 ${ }^{\mathrm{f} / \mathrm{f}} \mathrm{Alb}-\mathrm{Cre}$ ) developed severe hepatomegaly, followed by multiple tumors (Left panels). H\&E-staining of liver sections showed hepatocyte hypertrophy with inflammation in Atg $7^{\mathrm{f} / \mathrm{f}}$; Alb-Cre mice. Liver tumors in Atg $7^{\mathrm{f} / \mathrm{f}}$; Alb-Cre mice were diagnosed as hepatocellular adenoma (Right panels).

(Inami Y, et al: J Cell Biol, 2011; 193: 275-284 ${ }^{20)}$ )

\section{Conclusions}

The research about NAFLD/NASH is still in progress, and the recognition of the strategy for diagnose, treating or even preventing NAFLD/ NASH is still complicated. More investigations of the pathogenesis of NAFLD are necessary to develop useful biomarkers, effective therapy and prediction of prognosis of NAFLD/NASH.

\section{Acknowledgments}

I am grateful to all staff members of the department of gastroenterology in Juntendo University Faculty of Medicine that have supported my work over the years.

\section{Conflict of interests}

The author declares no conflict of interest associated with this manuscript.

\section{References}

1) Ludwig J, Viggiano TR, McGill DB, Oh BJ: Nonalcoholic steatohepatitis: Mayo Clinic experiences with a hitherto unnamed disease. Mayo Clin Proc, 1980; 55: 434-438.

2) Ikejima K, Watanabe S: Topics update in NAFLD: from bench side to clinics. Nihon Shokakibyo Gakkai Zasshi, 2014; 111: 4-13. (in Japanese)

3) Watanabe S, Yaginuma R, Ikejima K, Miyazaki A: Liver diseases and metabolic syndrome. J Gastroenterol, 2008; 43: 509-518.

4) Kabbany MN, Conjeevaram Selvakumar PK, Watt K, et $a l$ : Prevalence of nonalcoholic steatohepatitis-associated cirrhosis in the United States: an analysis of National Health and Nutrition Examination Survey Data. Am J Gastroenterol, 2017; 112: 581-587.

5) Watanabe S, Hashimoto E, Ikejima K, et al: Evidencebased clinical practice guidelines for nonalcoholic fatty liver disease/nonalcoholic steatohepatitis. Hepatol Res, 2015; 45: 363-377.

6) Watanabe S, Hashimoto E, Ikejima K, et al: Evidencebased clinical practice guidelines for nonalcoholic fatty liver disease/nonalcoholic steatohepatitis. J Gastroenterol, 2015; 50: 364-377.

7) Horie Y, Suzuki A, Kataoka E, et al: Hepatocyte-specific Pten deficiency results in steatohepatitis and hepatocellular carcinomas. J Clin Invest, 2004; 113: 1774-1783.

8) Day CP, James OF: Steatohepatitis: a tale of two "hits"? Gastroenterology, 1998; 114: 842-845. 
9) Tilg H, Moschen AR: Evolution of inflammation in nonalcoholic fatty liver disease: the multiple parallel hits hypothesis. Hepatology, 2010; 52: 1836-1846.

10) Morinaga M, Kon K, Saito H, et al: Sodium 4-phenylbutyrate prevents murine dietary steatohepatitis caused by trans-fatty acid plus fructose. J Clin Biochem Nutr, 2015; 57: 183-191.

11) Kon K, Ikejima K, Morinaga M, et al: L-carnitine prevents metabolic steatohepatitis in obese diabetic KK-Ay mice. Hepatol Res, 2017; 47: E44-54.

12) Ishikawa S, Ikejima $\mathrm{K}$, Yamagata $\mathrm{H}$, et al: CD1drestricted natural killer $\mathrm{T}$ cells contribute to hepatic inflammation and fibrogenesis in mice. J Hepatol, 2011; 54: 1195-1204

13) Hosoya S, Ikejima $K$, Takeda $K$, et al: Innate immune responses involving natural killer and natural killer $\mathrm{T}$ cells promote liver regeneration after partial hepatectomy in mice. Am J Physiol Gastrointest Liver Physiol, 2013; 304: G293-299.

14) Takashima S, Ikejima K, Arai K, et al: Glycine prevents metabolic steatohepatitis in diabetic $\mathrm{KK}-\mathrm{Ay}$ mice through modulation of hepatic innate immunity. Am J Physiol Gastrointest Liver Physiol, 2016; 311: G1105-
1113.

15) Ikejima K, Okumura K, Kon K, Takei Y, Sato N: Role of adipocytokines in hepatic fibrogenesis. J Gastroenterol Hepatol, 2007; 22 Suppl 1: S87-92.

16) Angin Y, Arslan N, Kuralay F: Leptin-to-adiponectin ratio in obese adolescents with nonalcoholic fatty liver disease. Turk J Pediatr, 2014; 56: 259-266.

17) Imajo K, Fujita K, Yoneda M, et al: Hyperresponsivity to low-dose endotoxin during progression to nonalcoholic steatohepatitis is regulated by leptin-mediated signaling. Cell metab, 2012; 16: 44-54.

18) Inami $Y$, Yamashina S, Izumi K, et al: Hepatic steatosis inhibits autophagic proteolysis via impairment of autophagosomal acidification and cathepsin expression. Biochem Biophys Res Commun, 2011; 412: 618-625.

19) Fukuo $Y$, Yamashina $S$, Sonoue $H$, et al: Abnormality of autophagic function and cathepsin expression in the liver from patients with non-alcoholic fatty liver disease. Hepatol Res, 2014; 44: 1026-1036.

20) Inami Y, Waguri S, Sakamoto A, et al: Persistent activation of Nrf2 through p62 in hepatocellular carcinoma cells. J Cell Biol, 2011; 193: 275-284. 